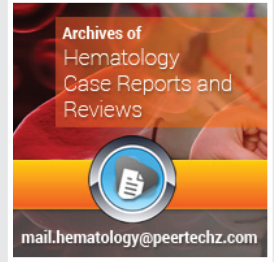

Clinical Group

\title{
Archives of Hematology Case Reports and Reviews
}

Maamar Kara², Omar Aitmokhtar ${ }^{1-3 *}$, Adel Azaza' ${ }^{2}$, Saber Seddiki ${ }^{2}$, Faiza Harbi $^{2}$, Arezki Sik ${ }^{2}$, Kheireddine Merad $^{2}$ and Salim Benkhedda ${ }^{2,3}$

'Department of cardiology, European hospital of Marseille, France

${ }^{2}$ Mustapha university hospital, Algiers, Algeria

${ }^{3}$ University of medicine of Algiers, Algeria

Dates: Received: 26 December, 2016; Accepted: 29 December, 2016; Published: 30 December, 2016

*Corresponding author: Omar Aitmokhtar, Hospital Européen, Department de Cardiologie, 2 rue Désiré clary 13002, Marseille, France, Tel: 0033615014988 ; E-Mail: aitmokhtar1@yahoo.fr

https://www.peertechz.com

\section{Abbreviations}

ACS: Acute Coronary Syndrome; AF: Atrial Fibrillation; DAPT: Dual Antiplatelet Therapy; INR: International Normalized Ratio; MI: Myocardial Infarction; PCI: Percutaneous Coronary Intervention; PIONEER AF-PCI: Open-Label, Randomized, Controlled, Multicenterstudy Exploring two Treatment Strategiesof Rivaroxaban and a Dose-Adjusted Oral Vitamink Antagonist Treatment Strategy in Subjectswith Atrial Fibrillation Who Undergo Percutaneous Coronary Intervention; TIMI: Thrombolysis In Myocardial Infarction; VKA: Vitamin K Antagonist

\section{Editorial}

Approximately $15 \%$ of $\mathrm{AF}$ patients have a history of myocardial infarction. Between $5-15 \%$ of them will require stenting at some point in their lives with the need for a triple therapy combining an oral anticoagulant, a P2Y12 Inhibitor and aspirin [1-3].

This combination requires careful evaluation of bleeding risk, stroke risk and the risk of acute coronary syndromes (ACS) to reduce the risk of major hemorrhage [4-6].

In this context, the PIONEER AF-PCI [7], trial was conducted to evaluate the effectiveness and safety of anticoagulation with rivaroxaban plus either one or two antiplatelet agents.

PIONEER AF-PCI included 2.124 patients with non-valvular $\mathrm{AF}$ (paroxysmal, persistent or permanent), oral anticoagulation for at least 3 months, and undergoing coronary angioplasty with stent placement. Within 3 days of the intervention, these patients were randomized to 3 antithrombotic treatments:
- Group 1: rivaroxaban at a dose of $15 \mathrm{mg}$ once daily (or a dose of $10 \mathrm{mg}$ once daily in moderate renal impairment with creatinine clearance of 30 to $50 \mathrm{ml}$ per minute) plus clopidogrel at a dose of $75 \mathrm{mg}$ once daily or ticagrelor at a dose of $90 \mathrm{mg}$ twice daily or prasugrel at adose of 10 mg once daily for 12 months.

- Group 2 : rivaroxaban at a dose of $2.5 \mathrm{mg}$ twice daily plus dual antiplatelet therapy (DAPT) with low-dose aspirin (75 to $100 \mathrm{mg}$ per day) and clopidogrel at a dose of 75 mg once daily (or ticagrelor at a dose of $90 \mathrm{mg}$ twice daily or prasugrel at a dose of $10 \mathrm{mg}$ once daily ) for a prespecified duration of 1,6 , or 12 months followed by rivaroxaban $15 \mathrm{mg} / \mathrm{d}$ (or $10 \mathrm{mg}$ / day in moderate renal insufficiency) in combination with aspirin until the end of the 12th month.

- Group 3 : Vitamin K Antagonist (VKA) warfarin once daily (with dose adjustment to achieve a targetINR of 2.0 to 3.0) plus DAPT with low-dose aspirin ( 75 to $100 \mathrm{mg}$ per day) and clopidogrel at a dose of $75 \mathrm{mg}$ once daily (or ticagrelor at a dose of $90 \mathrm{mg}$ twice daily or prasugrelat a dose of $10 \mathrm{mg}$ once daily for a prespecified duration of $\mathbf{1}$, 6 , or 12 months followed by an VKA in association with aspirin until the end of the 12th month.

In the majority of cases, investigators chose to prolong antiplatelet therapy for 12 months and the anti-P2Y 12 used was clopidogrel.

The primary safety end point was the occurrence of clinically significant bleeding (a composite of major bleeding or minor bleeding according to Thrombolysis in Myocardial Infarction (TIMI) criteria or bleeding requiring medical attention) during the treatment period.

Secondary end points included the incidence of each component of the primary safety end point, as well as the following efficacy end points: the occurrence of a major adverse cardiovascular event (a composite of death from cardiovascular causes, myocardial infarction, or stroke), each component of 
the major adverse cardiovascular event end point, and stent thrombosis.

The results show a marked reduction in bleeding in the two groups under rivaroxaban compared to patients in the third comparator group under VKA: $16.8 \%, 18 \%$ versus $26.7 \%$. The relative risks of bleeding in group 1 versus group 3 and group 2 versus group 3 were 0.59 (95\% CI [0.47-0.76]), and 0.63 (95\% CI $[0,50-0.80])$, respectively. $(\mathrm{p}<0.001)$.

Regarding the antithrombotic efficacy of the three protocols for prevention of thrombosis, the incidence of the composite criterion (CV death, MI, stroke) was not significantly different in the three groups: $6.5 \%, 5.6 \%$, and $6 \%$ respectively. The groups do not differ in terms of CV death, MI or stroke, considered independently.

The safety of protocols 1 or 2, compared to protocol 3, therefore seems to be gained. The question that remains unresolved is that of antithrombotic efficacy, and in particular with respect to stroke, since it is nevertheless their prevention that is aimed at anticoagulation in the AF.

The study does not establish, and even does not test frankly, non-inferiority in stroke prevention, strategies based on rivaroxaban compared to the standard of care that constitutes a VKA added to the dual antiplatelet therapy.

PIONEER was not sized for efficacy since patients at high risk of stroke were relatively few.

It should be noted that the doses of rivaroxaban used in PIONEER are below the recommended $20 \mathrm{mg}$ once daily in $\mathrm{AF}$ and that if the risk of stroke is not significantly different in group 1 versus 3 and in group 2 versus 3, the confidence intervals are considerable.

There are currently several ongoing studies on the benefits of new anticoagulants in patients with $\mathrm{AF}$ receiving a stent: REDUAL PCI with dabigatran, AUGUSTUS with apixaban, ENTRUST-AF-PCI with edoxaban.

However, PIONEER remains an important contribution to the data available in this field, which is in fact very limited
In conclusion, in AF patients undergoing PCI with placement of stents, the administration of either low-dose rivaroxaban plus a P2Y12 inhibitor for 12 months or verylow dose rivaroxaban plus DAPT for 1,6 , or 12 months was associated with a lower rate ofclinically significant bleeding than was standard therapy with a Vitamin K Antagonist plus DAPT for 1,6 , or 12 months. The three groups had similar efficacy rates, although thetrial was underpowered to evaluate stroke prevention.

\section{References}

1. Ruiz-Nodar JM, Marin F, Hurtado JA, Valencia J, Pinar E, et al. (2008) Anticoagulant and antiplatelet therapy use in 426 patients with atrial fibrillation undergoing percutaneous coronary intervention and stent implantation implications for bleeding risk and prognosis. J Am Coll Cardiol 51: 818-825. Link: https://goo.gl/Um9xpl

2. Hansen ML, Sorensen R, Clausen MT, Fog-Petersen ML, Raunso J, et al. (2010) Risk of bleeding with single, dual, or triple therapy with warfarin, aspirin, and clopidogrel in patients with atrial fibrillation. Arch Intern Med 170: 1433-1441. Link: https://goo.gl/I7qigX

3. Windecker S, Kolh P, Alfonso F, Collet JP, Cremer J, Falk V, et al. (2014) 2014 ESC/EACTS Guidelines on myocardial revascularization: The Task Force on Myocardial Revascularization of the European Society of Cardiology (ESC) and the European Association for Cardio-Thoracic Surgery (EACTS). Developed with the special contribution of the European Association of Percutaneous Cardiovascular Interventions (EAPCI). Eur Heart J 35: 2541 2619. Link: https://goo.gl/IPGXex

4. Dans AL, Connolly SJ, Wallentin L, Yang S, Nakamya J, et al. (2013) Concomitant use of antiplatelet therapy with dabigatran or warfarin in the Randomized Evaluation of Long-Term Anticoagulation Therapy (RE-LY) trial. Circulation 127: 634-640. Link: https://goo.gl/9leNbF

5. Vandvik PO, Lincoff AM, Gore JM, Gutterman DD, Sonnenberg FA, et al, (2012) Primary and secondary prevention of cardiovascular disease: Antithrombotic Therapy and Prevention of Thrombosis, 9th ed: AmericanCollege of Chest Physicians Evidence-Based Clinical Practice Guidelines. Chest 141: e637S668S. Link: https://goo.gl/SN2ao0

6. Rubboli A, Faxon DP, Juhani Airaksinen KE, Schlitt A, Marin F, et al. (2014) The optimal management of patients on oral anticoagulation undergoing coronary artery stenting. The 10th Anniversary Overview. ThrombHaemost 112: 1080-1087. Link: https://goo.gl/dOVam0

7. Gibson CM, Mehran R, Bode C, et al. (2016) Prevention of bleeding in patients with atrial fibrillation undergoing PCI. N Engl J Med 375:2423-2434. Link: https://goo.gl/ayulJU 